\title{
Discussion on the Design of Food Safety Subject Database Based on Big Data Testing
}

\author{
Kai Chen ${ }^{1,2}$, Heng Tao ${ }^{1, *}$, Jie Yu ${ }^{1,2}$, Miao Hao ${ }^{1,2}$, Hong Yang ${ }^{1}$ and Yajie Wang ${ }^{1,2}$ \\ ${ }^{1}$ Guizhou Academy of Sciences Big Data Co. LTD, Guiyang, Guizhou, 550000, China \\ ${ }^{2}$ Guizhou Academy of Testing and Analysis, Guiyang, Guizhou, 550000, China
}

\begin{abstract}
Food safety is a matter of national importance, and it is important to establish and improve a whole process food safety supervision system with high standard. Along with the advent of the era of big data, to achieve this goal requires not only the reform and optimization of the old regulatory approach needs to establish the idea of information-based supervision, and actively apply big data technology to food safety testing. In this paper, we introduce the current situation of food safety in society, analyse the shortcomings of current food safety supervision and the challenges faced in the era of big data, discuss the feasibility of using big data for food safety testing with big data technology as the core, and give the design idea of establishing a corresponding food safety subject database. This paper takes food safety testing as the foothold and innovatively combines big data with food safety testing, with a view to providing reference for food safety regulatory authorities and promoting the healthy development of the food industry.
\end{abstract}

\section{Introduction}

In recent years, China's rapid economic and social development, the country's economic strength continues to improve, and the people's demand for a better life is growing. Along with the renewal of technology, the food industry is also changing day by day, food production efficiency is greatly improved, food types are increasingly abundant, and the supply of products is abundant [1-2]. However, while the balance of food supply and demand is being met, food safety issues are becoming more and more prominent. The food industry has a long industrial chain, a large number of production links, a large number of small and medium-sized enterprises, and technological advances have made it difficult to detect many additives in food using traditional technical means, resulting in a period of high risk and contradictions in food safety. Strengthening food safety supervision has been a high priority for the Chinese government in recent years, while the general public's demand for food safety has become increasingly high. Although the Chinese government has adopted institutional reforms and other measures to strengthen supervision and enhance the ability of regulatory agencies to ensure the detection of food safety, the level of supervision has indeed steadily improved, and food safety problems have been effectively alleviated. However, traditional offline testing often requires a lot of human and material resources, and the testing efficiency is low. Along with the increasingly heavy task of food safety supervision, the difficulty of supervision has never been higher, and it is especially important to introduce the emerging big data technology to enhance the efficiency of testing and strengthen the supervisory capacity of testing institutions.

In the report of the 19th National Congress of the Chinese government, it is pointed out that "we are good at using Internet technology and information technology to carry out our work". This important instruction not only gives a blueprint for government departments to rely on big data, apply big data and develop big data, but also provides a brand new opportunity to improve the national governance system and enhance the national governance capacity. Under the guidance of this idea, the regulation of food safety has also begun to change to fast, accurate and efficient information-based regulation. The use of big data for food safety testing is also beginning to be In order to achieve a win-win situation for food manufacturers, consumers and food safety authorities, it is necessary to use big data technology to upgrade the level of food safety testing and to establish a sound food safety supervision system [3-5].

\section{Analysis of food safety issues in china}

\subsection{Current situation of food safety}

The food production and processing process goes through numerous stages such as breeding, production, processing, storage, transportation and sales, each of which may have potential food safety hazards caused by human factors.

There is an unavoidable drug contamination problem in the breeding process. Planting and breeding abuse or illegal use of hormones and prohibited drugs, food poisoning caused by pesticides, veterinary drugs [6].

\footnotetext{
*Corresponding author: taoheng@gzbdi.com
} 
These drug residues lay down unavoidable safety hazards for the subsequent food processing chain. The food safety and quality situation in the processing area is not optimistic, most of the food processing enterprises in China's production facilities and equipment are seriously outdated, the production environment is difficult to meet the requirements of food hygiene regulations and production standards, the health awareness of employees, quality and safety awareness are relatively weak, and the lack of the necessary means of field testing and quality assurance capabilities, coupled with some enterprises in order to reduce costs, the pursuit of efficiency and deliberately reduce Product quality, and even the use of toxic and harmful packaging materials, to the people's food safety needs have brought great damage. Food distribution is chaotic. Most of the food products in China are sold and distributed by individual businesses. Food storage and transportation equipment is old and it is difficult to guarantee safety measures in storage, transportation and sales. In addition, the use of excessive amounts of food additives is extremely serious, and in recent years there have been several major public incidents of food safety, causing great public panic. It can be seen that China's food safety problems are still relatively serious, and need to further enhance the technical means to strengthen supervision [7-8].

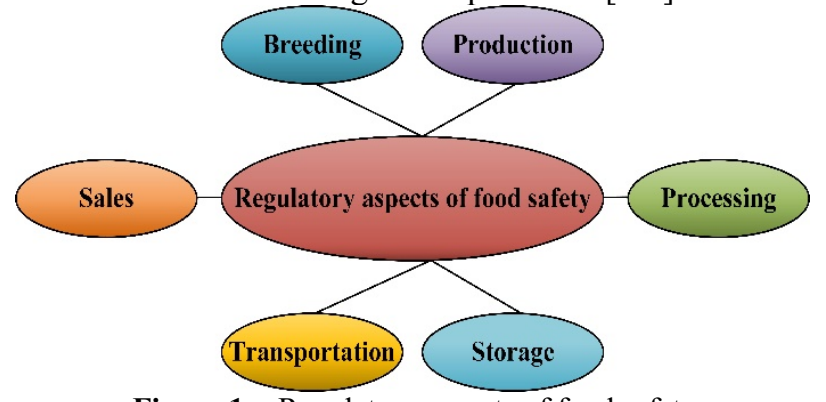

Figure 1. Regulatory aspects of food safety

\subsection{Shortcomings of current food safety supervision}

The main problems of current food regulation are imperfect construction of laws and regulations, low market access threshold, food standards are not uniform, food testing system is not perfect. Current regulatory authorities usually random testing and supervision of certain types of food by way of sampling or sampling tests, but due to the wide variety of food in the current market in China, the workload of sampling, the disadvantage is that it can not be a clear purpose sampling and key inspection items [9-10]. The shortcomings of the current food safety supervision can be summarized as follows:

(1) Food information lacks effective integration, making it difficult to capture early signs of dynamic changes in food safety in a timely manner.

(2) No quantitative standards for sampling and testing results, usually only a single pass rate and failure rate metric for sampling samples, unable to determine the risk value of the sampled batches.

(3) Timeliness is difficult to ensure that the test results lack of forward-looking prognosis, often only consider the current status of the food in the current link, can not be combined with other factors and comprehensive consideration and traceability.

The above analysis shows that the large regulatory responsibility and few regulatory tools have become bottlenecks to timely detection of food safety risks, the occasional occurrence of food safety accidents leading to increasing accountability, the difficulty of food sampling and the time consuming nature of testing makes it difficult to ensure food safety, and how to quickly and effectively detect food hazards is an important issue that needs to be addressed urgently today.

\subsection{The challenges of food safety faced in the era of big data}

Food safety supervision in the context of the big data era requires the introduction of emerging information technology, thus completing a disruptive innovation of the traditional regulatory mechanism. The root cause of food safety problems lies in the information asymmetry in the food market, and the information asymmetry caused by the lack of food safety information is an important factor in food safety problems. The existence of information barriers in government regulatory departments is not conducive to the effective development of food safety regulation. Therefore, in the process of changing government functions, it is important to fully recognize the importance of information technology, enhance the awareness of information services, actively develop and utilize information resources, optimize resource allocation, and promote healthy social and economic development. However, in this process, the following challenges are still faced.

(1) Data sharing. The regulatory big data of food safety needs to integrate the information of multiple government intelligent departments, and due to the lack of unified data standards, the data formats and standards of different departments are inconsistent, and there is a certain difficulty in data sharing. Traditional manual detection is also contrary to the expected goal of big data regulation. In addition, the data platform ports are not shared, and the variety of various food safety supervision data application platforms is not conducive to unified management.

(2) Data quality. Due to the large amount of data for food safety testing, the regulatory task is heavy, and the data of market entities are changing in real time, and manual testing affects the accuracy of the data to a certain extent. With the growth of the number of market entities, the amount of data continues to be expanded, but this type of data is mostly registration and approval information, such information lacks real-time dynamics. In addition, on the other hand, with the extension of the daily supervision surface, the data of comprehensive law enforcement supervision work has been increasing. However, due to the heavy regulatory tasks, detection information update interval is long, the updated regulatory information often exists lag.

(3) Data analysis. The current degree of utilization of food testing data is not high. The value of data is to use. Although the regulatory authorities have a huge amount 
of data on market players, the utilization rate of data is low, and there is little in-depth mining of information, which is still mainly based on manual experience, making it difficult to meet the current food safety requirements.

\section{Construction of food safety subject database}

The integration of food safety information resources mainly revolves around food safety testing data, centralizing the management of multiple information resources on food to improve regulatory efficiency and obtain real testing results, and to achieve optimal configuration of the food safety regulatory system. The essence of a food safety subject library is to integrate the information file resources of food testing, to significantly improve the difficulty of food safety supervision, to break the traditional information barriers, to enhance the easy access to food testing data, and to provide testing organizations with convenient access to information. From this point of view, the establishment of a food safety subject library helps the access and use of food safety information files. And in the current era of big data, where data has become an important strategic resource for China, the construction of a food safety subject library with big data testing at its core is particularly important. At present, in the actual work of food safety testing, there is often the problem of imperfect database construction that hinders the establishment of a food safety subject library. Therefore, the food safety subject library urgently needs the introduction of new technologies to strengthen the analysis and processing of food safety information [1112].

Database technology has now developed into a core technology of computer data processing and information management system. With the advent of the data age, the requirements for data processing are increasing, and the design of food safety libraries in the vast world of data will seem overwhelming if we rely on information technology alone without database technology. Database technology is an important support of information technology, which can be used to develop food safety supervision files by examining the internal structure, working principle and application status of existing databases. Database technology is an important support of information technology, which can solve the existing problems of data volume, content redundancy, storage space, etc. The database technology is an important support of information technology, which can solve the problems of large amount of data, redundant content, insufficient storage space and poor data dissemination and sharing in the integration of archival information resources, so that its knowledge and skills can be fully The research aims to solve the problems of data volume, redundant content, insufficient storage space, and poor data dissemination and sharing, so that their knowledge and skills can be fully utilized and the security, sharing and efficient retrieval of data can be guaranteed.

In the era of big data, the application of database technology in food safety supervision is targeted at food safety testing data. The use of this technology in integrating food safety information resources is mainly reflected in the following: First, by organizing and dividing food safety testing data in a unified manner, a food safety subject database is established according to specific rules, including a food safety information database and a food safety information data warehouse, respectively. Secondly, data management system and data mining system are used to design applications to supplement, modify, improve, and organize the data in the food safety information database. Finally, the database management application system is used to realize the analysis, processing, storage, and transmission of food safety information data. The use of database technology for a food safety subject library is the creation of a physical library where the file data formed at the level of each organization goes to another platform for unified management.

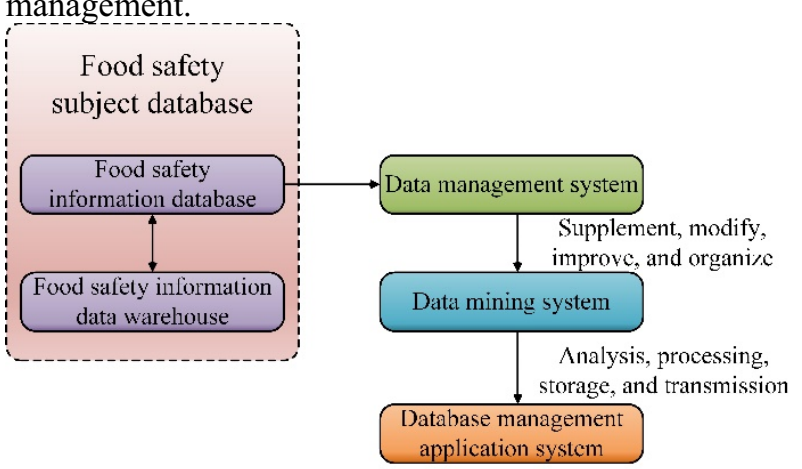

Figure 2. Food safety subject database

For the staff of a testing organization, the construction of a database can quickly provide them with a large amount of food safety information resources, and the staff of any testing organization can obtain the information they want on the platform built on this database. The use of database technology enables the construction of a food safety subject library, which can be enriched and updated in a timely manner. To this end, government departments at all levels should pay attention to the integration of food safety information resources, apply database technology to the supervision of food safety subject databases, and gradually deepen the integration of food safety information resources so that they are effectively organized and stored in a more holistic manner, while staff should strengthen the division of labour and cooperation to enrich the content of the subject databases.

\section{Conclusion}

The establishment of a food safety subject database can fully develop the value of food safety information, help make up for the deficiencies in food safety supervision work, improve the efficiency of testing agencies and regulatory departments, and help promote the harmonious development of society. At present, the establishment of a food safety theme bank has gradually become a social consensus, but there is still a need to further develop a clear system and standards to facilitate the development of a food safety subject database. In addition, the continuous development of big data technology can effectively promote the establishment of a food safety subject library, but it can also lead to some information 
security problems. Therefore, the development and design of food safety subject database still needs to be further promoted and improved. This paper discusses the feasibility of establishing a food safety subject database based on big data detection, with a view to promoting the development of the food industry and providing new ideas for regulatory authorities.

\section{Acknowledgement}

This work was supported by Guizhou Provincial Science and Technology Projects ([2018]5404 \& [2015]4005).

\section{References}

1. H. Bai and X. Liu, "Food nanotechnology and nano food safety," 2015 IEEE Nanotechnology Materials and Devices Conference (NMDC), Anchorage, AK, USA, 2015, pp. 1-4.

2. W. S. Boddie and L. Kun, "Health care, public health, and the food and agriculture critical infrastructures," in IEEE Engineering in Medicine and Biology Magazine, vol. 27, no. 6, pp. 54-58, NovemberDecember 2008.

3. H. Li, H. Xiao, T. Qiu and P. Zhou, "Food safety warning research based on internet public opinion monitoring and tracing," 2013 Second International Conference on Agro-Geoinformatics (AgroGeoinformatics), Fairfax, VA, USA, 2013, pp. 481484.

4. T. D. Ng and C. C. Yang, "A framework for harnessing public wisdom to ensure food safety," 2009 IEEE International Conference on Intelligence and Security Informatics, Richardson, TX, USA, 2009, pp. 185-187.

5. H. Lan, X. Chen and Y. Wu, "On food safety system construction from the perspective of supply chain," 2012 International Conference on Quality, Reliability, Risk, Maintenance, and Safety Engineering, Chengdu, China, 2012, pp. 1505-1507.

6. S. Li and J. Xu, "Research on food safety of China's food trade," Proceedings of International Conference on Information Systems for Crisis Response and Management (ISCRAM), Harbin, China, 2011, pp. 195-200.

7. H. Liu, J. Zheng, J. Zhang and L. Liu, "Measuring the Effect of Food Safety Incidents on China's Food Export: A Case Study on Aquatic Products," 2010 International Conference on Internet Technology and Applications, Wuhan, China, 2010, pp. 1-4.

8. Xiaobo Wang and Hongwu An, "The exploration on diversification of governmental administration tools-A case study on China domestic food safety administration," 2011 International Conference on Business Management and Electronic Information, Guangzhou, China, 2011, pp. 417-420.

9. W. Yu and S. Huang, "Traceability of Food Safety Based on Block Chain and RFID Technology," 2018 11th International Symposium on Computational
Intelligence and Design (ISCID), Hangzhou, China, 2018, pp. 339-342.

10. Chengping Ruan, Shiming Dai and Chengshen Ruan, "Study on the derived food safety management information system based on AJAX and Portal," 2011 International Conference on Multimedia Technology, Hangzhou, China, 2011, pp. 765-768.

11. Yan Ma, Jianjun Liu, Yongqiu Lin, Xiaopeng Cai and Zhen Liu, "Research and exploration of the Key Elements of Food Safety Data Analysis System based on the food safety traceability system," 2014 9th International Conference on Computer Science \& Education, Vancouver, BC, Canada, 2014, pp. 601605.

12. N. Kshetri, "Blockchain and the Economics of Food Safety," in IT Professional, vol. 21, no. 3, pp. 63-66, 1 May-June 2019. 